\title{
Existence of Solution for Non-Linear Functional Integral Equations of Two Variables in Banach Algebra
}

\author{
Hari M. Srivastava ${ }^{1,2, *(\mathbb{D}}$, Anupam Das ${ }^{3}$ and Bipan Hazarika ${ }^{3,4}$ and S. A. Mohiuddine ${ }^{5}$ (D) \\ 1 Department of Mathematics and Statistics, University of Victoria, Victoria, BC V8W 3R4, Canada \\ 2 Department of Medical Research, China Medical University Hospital, China Medical University, \\ Taichung 40402, Taiwan \\ 3 Department of Mathematics, Rajiv Gandhi University, Rono Hills, Doimukh 791112, Arunachal Pradesh, \\ India; math.anupam@gmail.com (A.D.); bh_rgu@yahoo.co.in (B.H.) \\ 4 Department of Mathematics, Gauhati University, Guwahati 781014, Assam, India \\ 5 Operator Theory and Applications Research Group, Department of Mathematics, Faculty of Science, King \\ Abdulaziz University, P.O. Box 80203, Jeddah 21589, Saudi Arabia; mohiuddine@gmail.com \\ * Correspondence: harimsri@math.uvic.ca
}

Received: 30 March 2019; Accepted: 5 May 2019; Published: 16 May 2019

\begin{abstract}
The aim of this article is to establish the existence of the solution of non-linear functional integral equations $x(l, h)=\left(U(l, h, x(l, h))+F\left(l, h, \int_{0}^{l} \int_{0}^{h} P(l, h, r, u, x(r, u)) d r d u, x(l, h)\right)\right) \times$ $G\left(l, h, \int_{0}^{a} \int_{0}^{a} Q(l, h, r, u, x(r, u)) d r d u, x(l, h)\right)$ of two variables, which is of the form of two operators in the setting of Banach algebra $C([0, a] \times[0, a]), a>0$. Our methodology relies upon the measure of noncompactness related to the fixed point hypothesis. We have used the measure of noncompactness on $C([0, a] \times[0, a])$ and a fixed point theorem, which is a generalization of Darbo's fixed point theorem for the product of operators. We additionally illustrate our outcome with the help of an interesting example.
\end{abstract}

Keywords: functional integral equations; Banach algebra; fixed point theorem; measure of noncompactness

MSC: (2010): 45G15; 47H10

\section{Introduction}

Many real-life problems in which we go over the investigation of various branches of mathematical physics, for example, gas kinetic theory, radiation, and neutron transportation, can be depicted and demonstrated by methods of non-linear functional integral equations (for example, we refer to [1-4]). Banaś and Lecko [5] introduced the concept of fixed points of product operators in Banach algebra. Dhage $[6,7]$ used the concept of the fixed point theorem to find the solution of functional integral equations in Banach algebra. Banaś and Olszowy [8] used the class of measures of noncompactness to obtain the existence of solutions of nonlinear integral equations in Banach algebra. Deepmala and Pathak [9] studied the existence of the solution of nonlinear functional integral equations of a single variable in Banach algebra $C[a, b]$ of all real-valued continuous functions on the interval $[a, b]$ equipped with the maximum norm. 
Kuratowski [10], in the year 1930, first introduced the idea of the measure of noncompactness (denoted by " $\alpha$ "). For any bounded subset $A$ of a metric space $X$,

$$
\alpha(A)=\inf \left\{\delta>0: A \subset \bigcup_{j=1}^{m} A_{j}, A_{j} \subset X, \operatorname{diam}\left(A_{j}\right)<\delta(j=1, \ldots, m), m \in \mathbb{N}\right\},
$$

where:

$$
\operatorname{diam}\left(A_{j}\right)=\sup \left\{d\left(a_{1}, a_{2}\right): a_{1}, a_{2} \in A_{j}\right\} .
$$

Using this idea, Darbo [11] exhibited a fixed point theorem that plays a very significant role in the finding of existence theorems. In the recent past, there have been a few fruitful endeavours to apply the idea of the measure of noncompactness in the investigation of the existence of solutions for various kinds of differential and integral equations, for example one can refer to [12-20].

In many physical problems, we come across nonlinear integral equations. The fixed point theory plays a significant role to obtain the solutions of such equations. Deepmala and Pathak, in [9], studied the following nonlinear functional integral equation, which can be considered as a particular case of many nonlinear functional integral equations that are applicable in mechanics, physics, economics, etc.,

$$
\begin{aligned}
x(t)= & \left(u(t, x(t))+f\left(t, \int_{0}^{t} p(t, s, x(s)) d s, x(\alpha(t))\right)\right) \\
& \times g\left(t, \int_{0}^{a} q(t, s, x(s)) d s, x(\beta(t))\right) \text { for } t \in[0, a] .
\end{aligned}
$$

The authors of [9] used the measure of noncompactness to obtain the existence of the solution of the integral Equation (1) in Banach algebra $C[0, a]$ with the help of the fixed point theorem.

Motivated by the work of [9], in this article, we study the solvability of non-linear functional integral equations of two variables, which we come across in various branches of nonlinear analysis. We consider an integral equation in the following form:

$$
\begin{aligned}
x(l, h)= & \left(U(l, h, x(l, h))+F\left(l, h, \int_{0}^{l} \int_{0}^{h} P(l, h, r, u, x(r, u)) d r d u, x(l, h)\right)\right) \\
& \times G\left(l, h, \int_{0}^{a} \int_{0}^{a} Q(l, h, r, u, x(r, u)) d r d u, x(l, h)\right) \text { for } l, h \in[0, a] .
\end{aligned}
$$

The right-hand side of the above integral equation that we are considering is the product of two functional operators involving integral operators and applying a fixed point theorem, which is a generalization of Darbo's fixed point theorem for the product of operators to check the existence of the solution of the integral equation in Banach algebra. It can be seen that Equation (2) is a generalization of Equation (1) in two variables. Here, we used a fixed point theorem associated with Darbo's condition of the measure of noncompactness in Banach algebra of continuous functions in $[0, a] \times[0, a]$ to establish the solvability of Equation (2). Furthermore, we used the modified homotopy perturbation analytic method to find the solution of Equation (2).

\section{Preliminaries}

Let $\mathbb{R}$ denote the set of real numbers, and write $\mathbb{R}_{+}=[0, \infty)$. Suppose $\bar{E}$ is a real Banach space with the norm $\|\cdot\|$, and let $X(\neq \phi) \subseteq \bar{E}$. The closure and convex closure of $X$ will be denoted by $\bar{X}$ and conv $X$, respectively. The convex closure of a set $X$ of points in the Euclidean plane or in a Euclidean space over the reals is the smallest convex set that contains $X$. A closed ball in $\bar{E}$ centred at $a$ and with radius $b$ is denoted by $B(a, b)$. In addition, we use the symbol $\mathcal{M}_{\bar{E}}$ to denote the family of all non-empty and bounded subsets of $\bar{E}$ and use $\mathcal{N}_{\bar{E}}$ to denote its subfamily consisting of all relatively compact sets. 
Definition 1. Let $X$ be a linear space over $\mathbb{R}$. A norm on $X$ is a function from $X$ to $\mathbb{R}_{+}$, commonly denoted $\|$. such that:

(N1) $\|x\| \geq 0$ and $\|x\|=0 \Longleftrightarrow x=0$;

(N2) $\| \alpha x||=|\alpha||| x||$;

(N3) $\|x+y\| \leq\|x\|+\|y\|$ for all $x, y \in X$ and $\alpha \in \mathbb{R}$.

The pair $(X,\|\|$.$) is called a normed space. A complete normed space is called a Banach space.$

Definition 2. An algebra $A$ is a vector space $A$ over a field $K$ such that for each ordered pair of elements $x, y \in A$, a unique product $x y \in A$ is defined with the properties:

(A1) $(x y) z=x(y z)$,

(A2) $x(y+z)=x y+x z$

(A3) $(x+y) z=x z+y z$,

(A4) $\alpha(x y)=(\alpha x) y=x(\alpha y)$ for all $x, y, z \in A$ and scalars $\alpha$.

A normed algebra $A$ is normed space, which is an algebra such that for all $x, y \in A$ :

$$
\|x y\| \leq\|x\|\|y\|
$$

and if $A$ has an identity $e$, then $\|e\|=1$.

A Banach algebra is a normed algebra that is complete, considered as a normed space.

The notion of the measure of noncompactness due to Banaś and Goebel [21] is as follows:

Definition 3. A function $\mu: \mathcal{M}_{\bar{E}} \rightarrow[0, \infty)$ is said to be a measure of noncompactness in $\bar{E}$ if:

(i) for all $X \in \mathcal{M}_{\bar{E}}$, we have that $\mu(X)=0$ implies that $X$ is precompact.

(ii) the family ker $\mu=\left\{X \in \mathcal{M}_{\bar{E}}: \mu(X)=0\right\}$ is non-empty, and ker $\mu \subset \mathcal{N}_{\bar{E}}$.

(iii) $X \subseteq Z \Longrightarrow \mu(X) \leq \mu(Z)$.

(iv) $\mu(\bar{X})=\mu(X)$.

(v) $\mu(\operatorname{conv} X)=\mu(X)$ where conv $X$ is the convex closure of set $X$.

(vi) $\mu(\lambda X+(1-\lambda) Z) \leq \lambda \mu(X)+(1-\lambda) \mu(Z)$ for $\lambda \in[0,1]$.

(vii) if $X_{n} \in \mathcal{M}_{\bar{E}}, X_{n}=\bar{X}_{n}, X_{n+1} \subset X_{n}$ for $n=1,2,3, \ldots$ and $\lim _{n \rightarrow \infty} \mu\left(X_{n}\right)=0$, then $\bigcap_{n=1}^{\infty} X_{n} \neq \phi$.

The family ker $\mu$ is called the kernel of measure $\mu$. Note that the intersection set $X_{\infty}$ from the above condition (vii) is a member of the family ker $\mu$. Since $\mu\left(X_{\infty}\right) \leq \mu\left(X_{n}\right)$ for any $n$, we deduce $\mu\left(X_{\infty}\right)=0$. Consequently, $X_{\infty} \in \operatorname{ker} \mu$.

For given subsets $X, Y$ in a Banach algebra $E$, the product $X Y$ defined by:

$$
X Y=\{x y: x \in X, y \in Y\} .
$$

In [8], Banaś and Olszowy defined the measure of noncompactness $\mu$ on the Banach algebra $E$, which satisfies condition $(m)$ if for arbitrary sets $X, Y \in \mathcal{M}_{E}$ such that:

$$
\mu(X Y) \leq\|X\| \mu(Y)+\|y\| \mu(X) .
$$

Deepmala and Pathak [9] used this concept of measure of noncompactness and obtained the existence of the solution of Equation (1).

Definition 4 ([21]). Let E be a Banach space. Consider a non-empty subset $X$ of $E$ and a continuous operator $T: X \rightarrow E$ transforming the bounded subset of $X$ to the bounded ones. We say that $T$ satisfies the Darbo condition with a constant $k$ with respect to measure $\mu$ provided $\mu(T Y) \leq k \mu(Y)$ for each $Y \in \mathcal{M}_{E}$ such that $Y \subset X$. If $k<1$, then $T$ is called a contraction with respect to $\mu$. 
Remark 1. The Darbo condition has many applications, particularly in fixed point theorems, which can be applied to check the existence of the solution of different types of integral, differential, and integro differential equations. The Darbo condition can be potentially applied to extend the linear space in the work of Shang [22]. The assumptions (1)-(4) of the next section have been utilized in the study of consensus problems (see [23,24]).

We recall the following important theorems:

Theorem 1 ([11]). Assume that $Z$ is a non-empty, closed, bounded, and convex subset of a Banach space $\bar{E}$. Let $S: Z \rightarrow Z$ be a continuous mapping. Suppose that there is a constant $k \in[0,1)$ such that:

$$
\mu(S M) \leq k \mu(M), M \subseteq Z .
$$

Then, S has a fixed point.

Theorem 2 ([8]). Suppose that $X$ is a non-empty, bounded, convex, and closed subset of a Banach algebra $E$, and the operators $P$ and $T$ transform continuously the set $X$ into $E$ such that $P(X)$ and $T(X)$ are bounded. Furthermore, suppose that the operator $S=P$.T transforms $X$ into itself. If $P$ and $T$ satisfy on the set $X$ the Darbo condition with respect to the measure of noncompactness $\mu$ with the constants $k_{1}$ and $k_{2}$, respectively, then $S$ satisfies on $X$ the Darbo condition with constant $\|P(X)\| k_{2}+\|T(X)\| k_{1}$. Particularly, if:

$$
\|P(X)\| k_{2}+\|T(X)\| k_{1}<1 \text {, }
$$

then $S$ is a contraction with respect to the measure of noncompactness $\mu$ and has at least one fixed point in $X$.

We consider the space $E=C([0, a] \times[0, a])$, which consists of the set of real-valued continuous functions on $[0, a] \times[0, a]$. It is obvious that $E$ is the vector space over the field of scalars $\mathbb{R}$ with the following operations:

$$
(x+y)(t, s)=x(t, s)+y(t, s)
$$

and:

$$
(\alpha x)(t, s)=\alpha x(t, s),
$$

where $x, y \in E, \alpha \in \mathbb{R}$ and $t, s \in[0, a]$. Since $x, y \in E$, i.e., both $x, y$ are real-valued continuous functions on $[0, a] \times[0, a]$ and the product of two real-valued continuous functions is also a real-valued continuous function, therefore $x y \in E$, where:

$$
(x y)(t, s)=x(t, s) y(t, s), t, s \in[0, a] .
$$

Let $z \in E$. For all $t, s \in[0, a]$,

$$
\begin{aligned}
((x y) z)(t, s) & =(x y)(t, s) z(t, s) \\
& =x(t, s) y(t, s) z(t, s) \\
& =x(t, s)(y z)(t, s) \\
& =(x(y z))(t, s) .
\end{aligned}
$$

Since $t, s$ are arbitrary, therefore $(x y) z=x(y z)$.

Similarly, it can be shown that:

$$
\begin{gathered}
x(y+z)=x y+x z \\
(x+y) z=x z+y z
\end{gathered}
$$

and:

$$
\alpha(x y)=(\alpha x) y=x(\alpha y) .
$$


Therefore $E$ is an algebra.

The space $E$ is also a normed space with the norm:

$$
\|x\|=\sup \{|x(l, h)|: l, h \in[0, a], a>0\}, x \in E .
$$

For all $x, y \in E$ and $l, h \in[0, a]$,

$$
|(x y)(l, h)|=|x(l, h) y(l, h)|=|x(l, h)||y(l, h)|
$$

and so:

$$
\sup _{l, h \in[0, a]}|(x y)(l, h)| \leq \sup _{l, h \in[0, a]}|x(l, h)| \sup _{l, h \in[0, a]}|y(l, h)|,
$$

i.e.,

$$
\|x y\| \leq\|x\|\|y\|
$$

Thus, $E$ is a normed algebra. Then:

Let $\left(x_{n}(t, s)\right)_{n=1}^{\infty}$ be a Cauchy sequence in $E$ where $x_{n}(t, s) \in \mathbb{R} \times \mathbb{R}$ for all $n \in \mathbb{N}$ and $t, s \in[0, a]$.

$$
\left\|x_{n}-x_{m}\right\| \rightarrow 0 \quad(n, m \rightarrow \infty) .
$$

Therefore, for all $t, s \in[0, a]$, we get:

$$
\left|x_{n}(t, s)-x_{m}(t, s)\right| \rightarrow 0 \quad(n, m \rightarrow \infty) .
$$

For fixed $t, s \in[0, a]$, the sequence $\left(x_{n}(t, s)\right)$ is a Cauchy sequence of real numbers, so it is a convergent sequence and converging to $x_{0}(t, s) \in E$ (say) as the limit of the continuous function is also continuous. Therefore, for all $t, s \in[0, a]$ :

$$
\left|x_{n}(t, s)-x_{0}(t, s)\right| \rightarrow 0 \quad(n, m \rightarrow \infty)
$$

which yields:

$$
\sup _{t, s \in[0, a]}\left|x_{n}(t, s)-x_{0}(t, s)\right| \rightarrow 0 \quad(n, m \rightarrow \infty)
$$

Thus:

$$
\left\|x_{n}-x_{0}\right\| \rightarrow 0 \quad(n \rightarrow \infty)
$$

which proves that $E$ is complete normed space. Hence, we conclude that the space $E$ has the Banach algebra structure.

Let $X$ be a fixed non-empty and bounded subset of $E=C([0, a] \times[0, a])$, and for $x \in X$ and $\epsilon>0$, the modulus of the continuity function (denoted by $\omega(x, \epsilon)$ ) is given by the formula:

$$
\omega(x, \epsilon)=\sup \{|x(l, h)-x(v, w)|: l, h, v, w \in[0, a],|l-v| \leq \epsilon,|h-w| \leq \epsilon\} .
$$

Further, we define:

$$
\omega(X, \epsilon)=\sup \{\omega(x, \epsilon): x \in X\}, \omega_{0}(X)=\lim _{\epsilon \rightarrow 0} \omega(x, \epsilon) .
$$

Similar to [5], it can be shown that the function $\omega_{0}(X)$ is a regular measure of non-compactness in the space $C([0, a] \times[0, a])$. Apart from this, it is easy to check that the measure $\omega_{0}(X)$ satisfies condition $(m)$. 


\section{Main Result}

In this section, we study the existence of solutions of the integral Equation (2). We consider the following assumptions:

(1) The functions $U:[0, a] \times[0, a] \times \mathbb{R} \rightarrow \mathbb{R}, F:[0, a] \times[0, a] \times \mathbb{R} \times \mathbb{R} \rightarrow \mathbb{R}$, and $G:[0, a] \times[0, a] \times$ $\mathbb{R} \times \mathbb{R} \rightarrow \mathbb{R}$ are continuous, and there exist nonnegative constants $L, M$ such that:

$$
|U(l, h, 0)| \leq L, \quad\left|F\left(l, h, M_{1}, 0\right)\right| \leq M \text { and }\left|F\left(l, h, M_{2}, 0\right)\right| \leq M,
$$

where $M_{1}, M_{2} \in \mathbb{R}$.

(2) Let $A_{i}:[0, a] \times[0, a] \rightarrow \mathbb{R}_{+}(i=1,2,3,4,5)$ be continuous functions such that:

$$
\begin{gathered}
\left|U\left(l, h, x_{1}\right)-U\left(l, h, x_{2}\right)\right| \leq A_{1}(l, h)\left|x_{1}-x_{2}\right|, \\
\left|F\left(l, h, y, x_{1}\right)-F\left(l, h, y, x_{2}\right)\right| \leq A_{2}(l, h)\left|x_{1}-x_{2}\right|, \\
\left|G\left(l, h, y_{1} x_{1}\right)-G\left(l, h, y_{2}\right)\right| \leq A_{3}(l, h)\left|x_{1}-x_{2}\right|, \\
\left|F\left(l, h, y_{1}, x\right)-F\left(l, h, y_{2}, x\right)\right| \leq A_{4}(l, h)\left|y_{1}-y_{2}\right|
\end{gathered}
$$

and:

$$
\left|G\left(l, h, y_{1}, x\right)-G\left(l, h, y_{2}, x\right)\right| \leq A_{5}(l, h)\left|y_{1}-y_{2}\right|,
$$

where $l, h \in[0, a]$ and $x, x_{1}, x_{2}, y, y_{1}, y_{2} \in \mathbb{R}$. Furthermore, let:

$$
K=\max \left\{A_{i}(l, h): i=1,2,3,4,5 ; l, h \in[0, a]\right\},
$$

where $K \geq 0$.

(3) The functions $P, Q$ are continuous functions from $[0, a] \times[0, a] \times[0, a] \times[0, a] \times \mathbb{R}$ to $\mathbb{R}$.

(4) Furthermore, $4 \alpha \beta<1$ for $\alpha=2 k, \beta=L+M$.

Theorem 3. Under the hypotheses (1)-(4), Equation (2) has at least one solution in $E=C(I \times I)$, where $I=[0, a]$.

Proof. Let us consider the operators $\hat{F}$ and $\hat{G}$ defined on $E$ by:

$$
(\hat{F} x)(l, h)=U(l, h, x(l, h))+F\left(l, h, \int_{0}^{l} \int_{0}^{h} P(l, h, r, u, x(r, u)) d r d u, x(l, h)\right)
$$

and:

$$
(\hat{G} x)(l, h)=G\left(l, h, \int_{0}^{a} \int_{0}^{a} Q(l, h, r, u, x(r, u)) d r d u, x(l, h)\right), \text { where } l, h \in[0, a] .
$$

From Assumptions (1)-(3), we get that $\hat{F}$ and $\hat{G}$ map $C(I \times I)$ into itself. Furthermore, let us define another operator $\hat{T}$ on $C(I \times I)$ as follows:

$$
\hat{T} x=(\hat{F} x)(\hat{G} x) .
$$

It is obvious that $\hat{T}$ maps $C(I \times I)$ into itself.

Let:

$$
I_{1}(x)=\int_{0}^{l} \int_{0}^{h} P(l, h, r, u, x(r, u)) d r d u
$$

and:

$$
I_{2}(x)=\int_{0}^{a} \int_{0}^{a} Q(l, h, r, u, x(r, u)) d r d u .
$$


Let $x \in C(I \times I)$ be fixed and $l, h \in I$. We get:

$$
\begin{aligned}
|(\hat{T} x)(l, h)|= & |(\hat{F} x)(l, h)| \cdot|(\hat{G} x)(l, h)| \\
= & \left|U(l, h, x(l, h))+F\left(l, h, I_{1}(x), x(l, h)\right)\right| \times\left|G\left(l, h, I_{2}(x), x(l, h)\right)\right| \\
\leq & \left(|U(l, h, x(l, h))-U(l, h, 0)|+|U(l, h, 0)|+\mid F\left(l, h, I_{1}(x), x(l, h)\right)\right. \\
& \left.-F\left(l, h, I_{1}(x), 0\right)|+| F\left(l, h, I_{1}(x), 0\right) \mid\right) \times\left(\mid G\left(l, h, I_{2}(x), x(l, h)\right)\right. \\
& \left.-G\left(l, h, I_{2}(x), 0\right)|+| G\left(l, h, I_{2}(x), 0\right) \mid\right) \\
\leq & \left(A_{1}(l, h)|x(l, h)|+L+A_{2}(l, h)|x(l, h)|+M\right) \times\left(A_{3}(l, h)|x(l, h)|+M\right) \\
\leq & (2 K\|x\|+L+M)(K\|x\|+M) \\
\leq & (2 K\|x\|+L+M)^{2} .
\end{aligned}
$$

Let $\alpha=2 k, \beta=L+M$. Then, we have:

$$
\begin{aligned}
& \|\hat{F} x\| \leq \alpha\|x\|+\beta, \\
& \|\hat{G} x\| \leq \alpha\|x\|+\beta
\end{aligned}
$$

and:

$$
\|\hat{T} x\| \leq(\alpha\|x\|+\beta)^{2}
$$

for $x \in C(I \times I)$.

From (3), we have that the operator $\hat{T}$ maps $B_{d} \subset C(I \times I)$ into $B_{d}$, where:

$$
B_{d}=\{x(l, h) \in I:\|x(l, h)\| \leq d\}
$$

for $d_{2} \leq d \leq d_{1}$, where:

$$
d_{1}=\frac{1-2 \alpha \beta-\sqrt{1-4 \alpha \beta}}{2 \alpha^{2}}
$$

and:

$$
d_{2}=\frac{1-2 \alpha \beta+\sqrt{1-4 \alpha \beta}}{2 \alpha^{2}} .
$$

Furthermore, we have:

$$
\left\|\hat{F} B_{d}\right\| \leq \alpha d+\beta
$$

and:

$$
\left\|\hat{G} B_{d}\right\| \leq \alpha d+\beta .
$$

Let $\epsilon>0$ be fixed and $x(l, h), y(l, h) \in B_{d}$ such that:

$$
\|x-y\| \leq \epsilon, \quad(l, h \in I) .
$$


Then, we have:

$$
\begin{aligned}
|(\hat{F} x)(l, h)-(\hat{F} y)(l, h)|= & \mid U(l, h, x(l, h))+F\left(l, h, I_{1}(x), x(l, h)\right) \\
& -U(l, h, y(l, h))-F\left(l, h, I_{1}(y), y(l, h)\right) \mid \\
\leq & |U(l, h, x(l, h))-U(l, h, y(l, h))| \\
& +\left|F\left(l, h, I_{1}(x), x(l, h)\right)-F\left(l, h, I_{1}(x), y(l, h)\right)\right| \\
& +\left|F\left(l, h, I_{1}(x), y(l, h)\right)-F\left(l, h, I_{1}(y), y(l, h)\right)\right| \\
\leq & A_{1}(l, h)|x(l, h)-y(l, h)|+A_{2}(l, h)|x(l, h)-y(l, h)| \\
& +A_{4}(l, h)\left|I_{1}(x)-I_{1}(y)\right| \\
\leq & 2 K\|x-y\| \\
& +K \int_{0}^{l} \int_{0}^{h}|P(l, h, r, u, x(r, u))-P(l, h, r, u, y(r, u))| d r d u \\
\leq & 2 K\|x-y\|+K a^{2} \omega(P, \epsilon),
\end{aligned}
$$

where:

$$
\omega(P, \epsilon)=\sup \left\{\begin{array}{c}
|P(l, h, r, u, x(r, u))-P(l, h, r, u, y(r, u))|: l, h, r, u \in I, \\
x, y \in[-d, d],\|x-y\|<\epsilon
\end{array}\right\} .
$$

Since $P$ is continuous, so it is uniformly continuous on the compact set $I \times I \times I \times I \times[-d, d]$; therefore:

$$
\omega(P, \epsilon) \rightarrow 0 \text { as } \epsilon \rightarrow 0 .
$$

Thus, $\hat{F}$ is continuous on $B_{d}$. Similarly, one can prove that $\hat{G}$ is continuous on $B_{d}$. Thus, we can conclude that $\hat{T}$ is continuous on $B_{d}$. 
Let us consider a non-empty subset $X$ of $B_{d}$ and $x \in X$. Then, for a fixed $\epsilon>0$ and $l_{1}, l_{2}, h_{1}, h_{2} \in I$ such that $l_{1} \leq l_{2}, h_{1} \leq h_{2}, l_{1}-l_{2} \leq \epsilon, h_{1}-h_{2} \leq \epsilon$, one obtains:

$$
\begin{aligned}
\mid & (\hat{F} x)\left(l_{2}, h_{2}\right)-(\hat{F} x)\left(l_{1}, h_{1}\right) \mid \\
= & \mid U\left(l_{2}, h_{2}, x\left(l_{2}, h_{2}\right)\right)+F\left(l_{2}, h_{2}, \int_{0}^{l_{2}} \int_{0}^{h_{2}} P\left(l_{2}, h_{2}, r, u, x(r, u)\right) d r d u, x\left(l_{2}, h_{2}\right)\right) \\
& -U\left(l_{1}, h_{1}, x\left(l_{1}, h_{1}\right)\right)-F\left(l_{1}, h_{1}, \int_{0}^{l_{1}} \int_{0}^{h_{1}} P\left(l_{1}, h_{1}, r, u, x(r, u)\right) d r d u, x\left(l_{1}, h_{1}\right)\right) \mid \\
\leq & \left|U\left(l_{2}, h_{2}, x\left(l_{2}, h_{2}\right)\right)-U\left(l_{2}, h_{2}, x\left(l_{1}, h_{1}\right)\right)\right|+\left|U\left(l_{2}, h_{2}, x\left(l_{1}, h_{1}\right)\right)-U\left(l_{1}, h_{1}, x\left(l_{1}, h_{1}\right)\right)\right| \\
& +\mid F\left(l_{2}, h_{2}, \int_{0}^{l_{2}} \int_{0}^{h_{2}} P\left(l_{2}, h_{2}, r, u, x(r, u)\right) d r d u, x\left(l_{2}, h_{2}\right)\right) \\
& -F\left(l_{2}, h_{2}, \int_{0}^{l_{1}} \int_{0}^{h_{1}} P\left(l_{1}, h_{1}, r, u, x(r, u)\right) d r d u, x\left(l_{2}, h_{2}\right)\right) \mid \\
& +\mid F\left(l_{2}, h_{2}, \int_{0}^{l_{1}} \int_{0}^{h_{1}} P\left(l_{1}, h_{1}, r, u, x(r, u)\right) d r d u, x\left(l_{2}, h_{2}\right)\right) \\
& -F\left(l_{1}, h_{1}, \int_{0}^{l_{1}} \int_{0}^{h_{1}} P\left(l_{1}, h_{1}, r, u, x(r, u)\right) d r d u, x\left(l_{2}, h_{2}\right)\right) \mid \\
& +\mid F\left(l_{1}, h_{1}, \int_{0}^{l_{1}} \int_{0}^{h_{1}} P\left(l_{1}, h_{1}, r, u, x(r, u)\right) d r d u, x\left(l_{2}, h_{2}\right)\right) \\
& +F\left(l_{1}, h_{1}, \int_{0}^{l_{1}} \int_{0}^{h_{1}} P\left(l_{1}, h_{1}, r, u, x(r, u)\right) d r d u, x\left(l_{2}, h_{2}\right)\right) \mid \\
& -F\left(l_{1}, h_{1}, \int_{0}^{l_{1}} \int_{0}^{h_{1}} P\left(l_{1}, h_{1}, r, u, x(r, u)\right) d r d u, x\left(l_{1}, h_{1}\right)\right) \mid \\
\leq & A_{1}(l, h)\left|x\left(l_{2}, h_{2}\right)-x\left(l_{1}, h_{1}\right)\right|+\left|U\left(l_{2}, h_{2}, x\left(l_{1}, h_{1}\right)\right)-U\left(l_{1}, h_{1}, x\left(l_{1}, h_{1}\right)\right)\right| \\
+ & A_{4}(l, h)\left|\int_{0}^{l_{2}} \int_{0}^{h_{2}} P\left(l_{2}, h_{2}, r, u, x(r, u)\right) d r d u-\int_{0}^{l_{1}} \int_{0}^{h_{1}} P\left(l_{1}, h_{1}, r, u, x(r, u)\right) d r d u\right| \\
& \mid F\left(l_{2}, h_{2}, \int_{0}^{l_{1}} \int_{0}^{h_{1}} P\left(l_{1}, h_{1}, r, u, x(r, u)\right) d r d u, x\left(l_{2}, h_{2}\right)\right) \\
& \mid
\end{aligned}
$$

which yields:

$$
\begin{aligned}
& \left|(\hat{F} x)\left(l_{2}, h_{2}\right)-(\hat{F} x)\left(l_{1}, h_{1}\right)\right| \leq 2 K\left|x\left(l_{2}, h_{2}\right)-x\left(l_{1}, h_{1}\right)\right| \\
& +\left|U\left(l_{2}, h_{2}, x\left(l_{1}, h_{1}\right)\right)-U\left(l_{1}, h_{1}, x\left(l_{1}, h_{1}\right)\right)\right| \\
& +K \mid \int_{0}^{l_{2}} \int_{0}^{h_{2}} P\left(l_{2}, h_{2}, r, u, x(r, u)\right) d r d u
\end{aligned}
$$




$$
\begin{aligned}
& -\int_{0}^{l_{1}} \int_{0}^{h_{1}} P\left(l_{1}, h_{1}, r, u, x(r, u)\right) d r d u \mid \\
& +\mid F\left(l_{2}, h_{2}, \int_{0}^{l_{1}} \int_{0}^{h_{1}} P\left(l_{1}, h_{1}, r, u, x(r, u)\right) d r d u, x\left(l_{2}, h_{2}\right)\right) \\
& -F\left(l_{1}, h_{1}, \int_{0}^{l_{1}} \int_{0}^{h_{1}} P\left(l_{1}, h_{1}, r, u, x(r, u)\right) d r d u, x\left(l_{2}, h_{2}\right)\right) \mid .
\end{aligned}
$$

Let:

$$
\begin{gathered}
\omega(U, \epsilon)=\sup \left\{\begin{array}{c}
\left|U\left(l_{2}, h_{2}, x\left(l_{2}, h_{2}\right)\right)-U\left(l_{1}, h_{1}, x\left(l_{1}, h_{1}\right)\right)\right|: l_{1}, l_{2}, h_{1}, h_{2} \in I, \\
\left|l_{2}-l_{1}\right| \leq \epsilon,\left|h_{2}-h_{1}\right| \leq \epsilon, x \in[-d, d]
\end{array}\right\}, \\
\omega(P, \epsilon)=\sup \left\{\begin{array}{c}
\left|P\left(l_{2}, h_{2}, r, u, x(r, u)\right)-P\left(l_{1}, h_{1}, r, u, x(r, u)\right)\right|: l_{1}, l_{2}, h_{1}, h_{2}, r, u \in I, \\
\left|l_{2}-l_{1}\right| \leq \epsilon,\left|h_{2}-h_{1}\right| \leq \epsilon, x \in[-d, d]
\end{array}\right\}, \\
\bar{k}=\sup \{|P(l, h, r, u, x(r, u))|: l, h, r, u \in I, x \in[-d, d]\}
\end{gathered}
$$

and:

$$
\omega(F, \epsilon)=\sup \left\{\begin{array}{c}
\left|F\left(l_{2}, h_{2}, z, x\left(l_{2}, h_{2}\right)\right)-F\left(l_{1}, h_{1}, z, x\left(l_{1}, h_{1}\right)\right)\right|: l_{1}, l_{2}, h_{1}, h_{2} \in I, \\
\left|l_{2}-l_{1}\right| \leq \epsilon,\left|h_{2}-h_{1}\right| \leq \epsilon, x \in[-d, d], z \in\left[-\bar{k} a^{2}, \bar{k} a^{2}\right]
\end{array}\right\}
$$

Furthermore:

$$
\begin{aligned}
& \left|\int_{0}^{l_{2}} \int_{0}^{h_{2}} P\left(l_{2}, h_{2}, r, u, x(r, u)\right) d r d u-\int_{0}^{l_{1}} \int_{0}^{h_{1}} P\left(l_{1}, h_{1}, r, u, x(r, u)\right) d r d u\right| \\
& \leq\left|\int_{0}^{l_{2}} \int_{0}^{h_{2}}\left(P\left(l_{2}, h_{2}, r, u, x(r, u)\right)-P\left(l_{1}, h_{1}, r, u, x(r, u)\right)\right) d r d u\right| \\
& +\left|\int_{l_{1}}^{l_{2}} \int_{h_{1}}^{h_{2}} P\left(l_{1}, h_{1}, r, u, x(r, u)\right) d r d u\right| \\
& \leq a^{2} \omega(P, \epsilon)+\bar{k} \epsilon^{2} .
\end{aligned}
$$

Therefore:

$$
\begin{aligned}
\left|(\hat{F} x)\left(l_{2}, h_{2}\right)-(\hat{F} x)\left(l_{1}, h_{1}\right)\right| \leq & 2 K\left|x\left(l_{2}, h_{2}\right)-x\left(l_{1}, h_{1}\right)\right|+\omega(U, \epsilon) \\
& +K\left(a^{2} \omega(P, \epsilon)+\bar{k} \epsilon^{2}\right)+\omega(F, \epsilon) .
\end{aligned}
$$

This gives:

$$
\omega(\hat{F} x, \epsilon) \leq 2 K \omega(x, \epsilon)+\omega(U, \epsilon)+K\left[a^{2} \omega(P, \epsilon)+\bar{k} a^{2}\right]+\omega(F, \epsilon) .
$$

Since $U$ and $F$ are continuous on $I \times I \times \mathbb{R}$ and $I \times I \times \mathbb{R} \times \mathbb{R}$, respectively, therefore we get:

$$
\omega(U, \epsilon) \rightarrow 0, \omega(P, \epsilon) \rightarrow 0 \text { and } \omega(F, \epsilon) \rightarrow 0 \text { as } \epsilon \rightarrow 0 .
$$

Thus:

$$
\omega_{0}(\hat{F} X) \leq 2 K \omega_{0}(X)
$$

Similarly, we can show that:

$$
\omega_{0}(\hat{G} X) \leq 2 K \omega_{0}(X)
$$


From (4)-(7) and Theorem 2 (for the details of this theorem, we refer to [8]), we get that $\hat{T}$ satisfies the Darbo condition on $B_{d}$ with respect to measure $\omega_{0}$ with constant:

$$
\begin{aligned}
2 K(\alpha d+\beta)+2 K(\alpha d+\beta) & =4 K(\alpha d+\beta) \\
& =4 K\left(\alpha d_{1}+\beta\right) \\
& =4 K\left[\alpha\left(\frac{1-2 \alpha \beta-\sqrt{1-4 \alpha \beta}}{2 \alpha^{2}}\right)+\beta\right] \\
& =2 K\left(\frac{1-\sqrt{1-4 \alpha \beta}}{\alpha}\right) \\
& <1 .
\end{aligned}
$$

This implies that $\hat{T}$ is a contraction operator on $B_{d}$ with respect to $\omega_{0}$. Thus, by Theorem 2 , we have that $\hat{T}$ has at least one fixed point in $B_{d}$. Hence, Equation (2) has at least one solution in $B_{d} \subset C([0, a] \times[0, a])$. This completes the proof.

\section{An Illustrative Example}

We construct the following example to illustrate the obtained result in the previous section.

Example 1. Consider the following integral equation:

$$
x(l, h)=\left(\frac{1}{6} \cos \left(\frac{l+h}{2}\right)+\frac{1}{9} \int_{0}^{l} \int_{0}^{h} \frac{r u e^{-l h}}{3+x^{2}(r, u)} d r d u\right)\left(\frac{1}{8} \int_{0}^{1} \int_{0}^{1} \frac{l h}{6+|x(r, u)|} d r d u\right)
$$

for $l, h \in[0,1]=I$. Here, we have:

$$
\begin{gathered}
U(l, h, x(l, h))=\frac{1}{6} \cos \left(\frac{l+h}{2}\right), \\
F(l, h, y, x(l, h))=\frac{y}{9}, \\
G(l, h, y, x(l, h))=\frac{y}{8}, \\
P(l, h, r, u, x(r, u))=\frac{r u e^{-l h}}{3+x^{2}(r, u)}, \\
Q(l, h, r, u, x(r, u))=\frac{l h}{6+|x(r, u)|}
\end{gathered}
$$

and $a=1 ; x, y \in \mathbb{R}$.

It is obvious that all the functions $U, F, G, P$, and $Q$ are continuous. We have:

$$
\begin{gathered}
\left|U\left(l, h, x_{1}\right)-U\left(l, h, x_{2}(l, h)\right)\right|=0 .\left|x_{1}(l, h)-x_{2}(l, h)\right|, \\
\left|F\left(l, h, y, x_{1}(l, h)\right)-F\left(l, h, y, x_{2}(l, h)\right)\right|=0 .\left|x_{1}(l, h)-x_{2}(l, h)\right|, \\
\left|G\left(l, h, y, x_{1}(l, h)\right)-G\left(l, h, y, x_{2}(l, h)\right)\right|=0 .\left|x_{1}(l, h)-x_{2}(l, h)\right|, \\
\left|F\left(l, h, y_{1}, x(l, h)\right)-F\left(l, h, y_{2}, x(l, h)\right)\right|=\frac{1}{9}\left|y_{1}-y_{2}\right|,
\end{gathered}
$$

and:

$$
\left|G\left(l, h, y_{1}, x(l, h)\right)-G\left(l, h, y_{2}, x(l, h)\right)\right|=\frac{1}{8}\left|y_{1}-y_{2}\right|
$$


It follows that:

$$
A_{1}(l, h)=A_{2}(l, h)=A_{3}(l, h)=0, A_{4}(l, h)=\frac{1}{9} \text { and } A_{5}(l, h)=\frac{1}{8} .
$$

Consequently, we get $K=\frac{1}{8}$.

Furthermore,

$$
\begin{gathered}
|U(l, h, 0)| \leq \frac{1}{6} \\
\left|F\left(l, h, y_{1}, 0\right)\right| \leq \frac{1}{27 e^{2}} \approx \frac{1}{198.29}
\end{gathered}
$$

and:

$$
\left|G\left(l, h, y_{2}, 0\right)\right| \leq \frac{1}{48}
$$

Thus:

$$
M=\frac{1}{48}, L=\frac{1}{6}
$$

and:

$$
4 \alpha \beta=\frac{9}{48}<1 .
$$

Hence, all the assumption from (1)-(4) are satisfied. Thus, by applying Theorem 3, we conclude that Equation (8) has at least one solution in the Banach algebra $C([0,1] \times[0,1])$.

\section{An Iterative Algorithm Created by a Coupled Semi-Analytic Method to Find the Solution of the Integral Equation}

To find an approximation of solution for Equation (8), we make an iterative algorithm by a coupled method created by modified homotopy perturbation and the Adomian decomposition method in the case of two-dimensional functions. Applications of the modified homotopy perturbation method to solve nonlinear integral equations, nonlinear singular integral equations, and nonlinear differential equations can be seen in [25-27], respectively. The Adomian decomposition method to solve physical problems was used in [28] and also to solve integro-differential equations system in [29]. However, in this article, we introduce a modified homotopy perturbation method in terms of a function with two variables, and for simplification of nonlinear terms, we use the Adomian decomposition method in the suitable form; therefore, we make an effective algorithm by the above process. Equation (8) can be shown in a general form of the two-dimensional nonlinear problem:

$$
A(x(l, h))-f(l, h)=0
$$

with $(l, h) \in I \times I$, where $A$ is a general nonlinear operator and $f$ is a known analytic function. Similar to [26,27], we divide the general operator $A$ into two nonlinear operators as $M_{1}$ and $M_{2}$. Of course, $M_{1}$ or $M_{2}$ can be linear operators in the special case that also $f$ is converted to $f_{1}$ and $f_{2}$ functions; in other words, we have:

$$
M_{1}(x(l, h))-f_{1}(l, h)+M_{2}(x(l, h))-f_{2}(l, h)=0 .
$$

A modified homotopy perturbation for the above problem can be introduced as follows:

$$
H(u(l, h), p)=M_{1}(u(l, h))-f_{1}(l, h)+p\left[M_{2}(u(l, h))-f_{2}(l, h)\right]=0, p \in[0,1],
$$

where $p$ is an embedding parameter and $u$ is an approximation of $x$. According to the variations of $p=0$ to $p=1$, it can be observed that $M_{1}(u(l, h))=f_{1}(l, h)$ to $A(u(l, h))=f(l, h)$. This implies that for $p=1$ in (9), we get the solution of (5). 
We consider the above solution as the series:

$$
x(l, h) \simeq u(l, h)=\sum_{k=0}^{\infty} p^{k} u_{k}(l, h)
$$

and:

$$
x(l, h)=\lim _{p \rightarrow 1} u(l, h) .
$$

To solve Equation (8), $M_{1}, M_{2}$ and $f$ can be defined as follows:

$$
\begin{gathered}
M_{1}(x(l, h))=x(l, h), \\
M_{2}(x(l, h))=-\left(\frac{1}{6} \cos \left(\frac{l+h}{2}\right)+\frac{1}{9} \int_{0}^{l} \int_{0}^{h} \frac{r u e^{-l h}}{3+x^{2}(r, u)} d r d u\right)\left(\frac{1}{8} \int_{0}^{1} \int_{0}^{1} \frac{l h}{6+|x(r, u)|} d r d u\right),
\end{gathered}
$$

and:

$$
f(l, h)=f_{1}(l, h)+f_{2}(l, h) .
$$

Since in (8) $f(l, h)=0$, therefore $f_{1}(l, h)=f_{2}(l, h)=0$. From (9)-(14), we have:

$$
\begin{aligned}
& \sum_{k=0}^{\infty} p^{k} u_{k}(l, h) \\
& -p\left(\frac{1}{6} \cos \left(\frac{l+h}{2}\right)+\frac{1}{9} \int_{0}^{l} \int_{0}^{h} \frac{r u e^{-l h}}{3+\left(\sum_{k=0}^{\infty} p^{k} u_{k}(r, u)\right)^{2}} d r d u\right) \\
& \left(\frac{1}{8} \int_{0}^{1} \int_{0}^{1} \frac{l h}{6+\left|\sum_{k=0}^{\infty} p^{k} u_{k}(r, u)\right|} d r d u\right)=0 .
\end{aligned}
$$

Now, we use Adomain polynomials for simplicity for the nonlinear terms:

$$
\frac{1}{6} \cos \left(\frac{l+h}{2}\right)+\frac{1}{9} \int_{0}^{l} \int_{0}^{h} \frac{r u e^{-l h}}{3+\left(\sum_{k=0}^{\infty} p^{k} u_{k}(r, u)\right)^{2}} d r d u=\sum_{k=0}^{\infty} p^{k} A_{k}(l, h)
$$

and:

$$
\frac{1}{8} \int_{0}^{1} \int_{0}^{1} \frac{l h}{6+\left|\sum_{k=0}^{\infty} p^{k} u_{k}(r, u)\right|} d r d u=\sum_{k=0}^{\infty} p^{k} \hat{A}_{k}(l, h),
$$

where the Adomain polynomials are given by:

$$
A_{k}(l, h)=\frac{1}{k !}\left[\frac{d^{k}}{d p^{k}}\left\{\frac{1}{6} \cos \left(\frac{l+h}{2}\right)+\frac{1}{9} \int_{0}^{l} \int_{0}^{h} \frac{r u e^{-l h}}{3+\left(\sum_{k=0}^{\infty} p^{k} u_{k}(r, u)\right)^{2}} d r d u\right\}\right]_{p=0}
$$

and:

$$
\hat{A}_{k}(l, h)=\frac{1}{k !}\left[\frac{d^{k}}{d p^{k}}\left\{\frac{1}{8} \int_{0}^{1} \int_{0}^{1} \frac{l h}{6+\left|\sum_{k=0}^{\infty} p^{k} u_{k}(r, u)\right|} d r d u\right\}\right]_{p=0}
$$


Therefore, we have:

$$
\sum_{k=0}^{\infty} p^{k} u_{k}(l, h)-p\left\{\sum_{k=0}^{\infty} p^{k} A_{k}(l, h)\right\}\left\{\sum_{k=0}^{\infty} p^{k} \hat{A}_{k}(l, h)\right\}=0 .
$$

By rearranging the terms in powers in $p$ of (15) and using modified homotopy perturbation (9), the coefficients of $p$ powers must be equal to zero, so we obtain an iterative algorithm (Algorithm 1) to solve for the numerical solution of Equation (8).

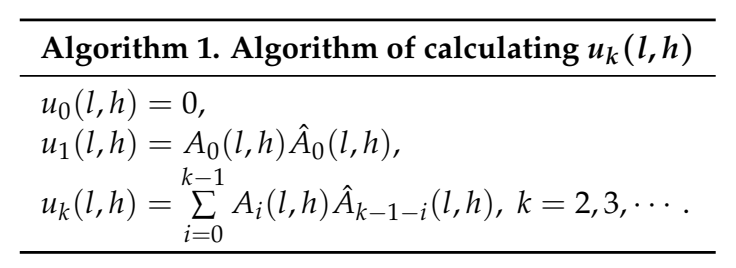

Calculating the sequence $\left\{u_{0}(l, h), u_{1}(l, h), \ldots\right\}$, we can obtain a closed form of the solution for (8) using the above algorithm.

We compute the Adomain polynomial for $k=0$,

$$
\begin{aligned}
& A_{0}(l, h) \\
& =\frac{1}{6} \cos \left(\frac{l+h}{2}\right)+\frac{1}{9} \int_{0}^{l} \int_{0}^{h} \frac{r u e^{-l h}}{3+u_{0}^{2}(r, u)} d r d u \\
& =\frac{1}{6} \cos \left(\frac{l+h}{2}\right)+\frac{l^{2} h^{2} e^{-l h}}{108}
\end{aligned}
$$

and:

$$
\hat{A}_{0}(l, h)=\frac{1}{8} \int_{0}^{1} \int_{0}^{1} \frac{l h}{6+\left|u_{0}(r, u)\right|} d r d u=\frac{l h}{48} .
$$

Therefore, we obtain by the algorithm:

$$
u_{1}(l, h)=\left\{\frac{1}{6} \cos \left(\frac{l+h}{2}\right)+\frac{l^{2} h^{2} e^{-l h}}{108}\right\} \frac{l h}{48} .
$$

We use (10) to approximate $x(l, h)$ by a few term of $u_{k}(l, h)$ as follows:

$$
x_{1}(l, h)=u_{0}(l, h)+u_{1}(l, h)=\left\{\frac{1}{6} \cos \left(\frac{l+h}{2}\right)+\frac{l^{2} h^{2} e^{-l h}}{108}\right\} \frac{l h}{48} .
$$

\section{Conclusions}

In our present investigation, we have established the existence of the solution of a functional integral equation of two variables, which is of the form of the product of two operators in the Banach algebra $C([0, a] \times[0, a]), a>0$ and illustrated our results with the help of an example. We also constructed an iteration algorithm to get the solution of Equation (8). Further, one can solve Equation (8) using different numerical, as well as analytical methods in the setting of Banach sequence spaces and Banach algebra. Moreover, due our existence theorem for Equation (8) of two variables, we therefore conclude that our existence result is more general than the one obtained earlier by Deepmala and Pathak [9]. 
Author Contributions: All authors contributed equally in this work.

Funding: This research received no external funding.

Conflicts of Interest: The authors declare no conflict of interest.

\section{References}

1. Chandrasekhar, S. Radiative Transfer; Oxford University Press: London, UK, 1950.

2. Hu, S.; Khavanin, M.; Zhuang, W. Integral equations arising in the kinetic theory of grass. Appl. Anal. 1989, 34, 261-266. [CrossRef]

3. Kelly, C.T. Approximation of solution of some quadratic integral equations in transport theory. J. Integral Equ. 1982, 4, 221-237.

4. Zabrejko, P.P.; Koshelev, A.I.; Krasnosel'skii, M.A.; Mikhlin, S.G.; Rakovshchik, L.S.; Stetsenko, V.J. Integral Equations; Nauka: Moscow, Russia, 1968.

5. Banaś, J.; Lecko, M. Fixed points of the product of operators in Banach algebra. Panamer Math. J. 2002, 12, 101-109.

6. Dhage, B.C. A flxed point theorem in Banach algebras with applications to functional integral equations. Kyungpook Math. J. 2004, 44, 145-155.

7. Dhage, B.C. On a flxed point theorem in Banach algebras with applications. Appl. Math. Lett. 2005, 18, 273-280. [CrossRef]

8. Banaś, J.; Olszowy, L. On a class of measure of noncompactness in Banach algebras and their application to nonlinear integral equations. J. Anal. Appl. 2009, 28, 1-24. [CrossRef]

9. Pathak, H.K. A study on some problems on existence of solutions for nonlinear functional-integral equations. Acta Math. Sci. 2013, 33, 1305-1313.

10. Kuratowski, K. Sur les espaces complets. Fund. Math. 1930, 15, 301-309. [CrossRef]

11. Darbo, G. Punti uniti in trasformazioni a codominio non compatto. Rend. Sem. Mater. Univ. Padova 1955, 24, 84-92.

12. Alotaibi, A.; Mursaleen, M.; Mohiuddine, S.A. Application of measure of noncompactness to infinite system of linear equations in sequence spaces. Bull. Iran. Math. Soc. 2015, 41, 519-527.

13. Aghajani, A.; Allahyari, R.; Mursaleen, M. A generalization of Darbo's theorem with application to the solvability of systems of integral equations. J. Comput. Appl. Math. 2014, 260, 68-77. [CrossRef]

14. Arab, R.; Allahyari, R.; Haghighi, A.S. Existence of solutions of infinite systems of integral equations in two variables via measure of noncompactness. Appl. Math. Comput. 2014, 246, 283-291. [CrossRef]

15. Darwish, M.A. On quadratic integral equation of fractional orders. J. Math. Anal. Appl. 2005, 311, 112-119. [CrossRef]

16. Mishra, L.N.; Sen, M.; Mohapatra, R.N. On existence theorems for some generalized nonlinear functional-integral equations with applications. Filomat 2017, 31, 2081-2091.

17. Mohiuddine, S.A.; Srivastava, H.M.; Alotaibi, A. Application of measures of noncompactness to the infinite system of second-order differential equations in $l_{p}$ spaces. Adv. Differ. Equ. 2016, 2016, 317. [CrossRef]

18. Mursaleen, M.; Mohiuddine, S.A. Applications of measures of noncompactness to the infinite system of differential equations in $l_{p}$ spaces. Nonlinear Anal. 2012, 75, 2111-2115. [CrossRef]

19. Mursaleen, M.; Rizvi, S.M.H. Solvability of infinite systems of second order differential equations in $c_{0}$ and $\ell_{1}$ by Meir-Keeler condensing operators. Proc. Am. Math. Soc. 2016, 144, 4279-4289. [CrossRef]

20. Srivastava, H.M.; Das, A.; Hazarika, B.; Mohiuddine, S.A. Existence of solutions of infinite systems of differential equations of general order with boundary conditions in the spaces $c_{0}$ and $l_{1}$ via the measure of noncompactness. Math. Meth. Appl. Sci. 2018, 41, 3558-3569. [CrossRef]

21. Banaś, J.; Goebel, K. Measure of Noncompactness in Banach Spaces, Lecture Notes in Pure and Applied Mathematics; Marcel Dekker: New York, NY, USA, 1980; Volume 60.

22. Shang, Y. Subspace confinement for switched linear systems. Forum Math. 2017, 29, 693-699. [CrossRef]

23. Shang, Y. Fixed-time group consensus for multi-agent systems with non-linear dynamics and uncertainties. IET Control Theory Appl. 2018, 12, 395-404. [CrossRef]

24. Shang, Y. Resilient consensus of switched multi-agent systems. Syst. Control Lett. 2018, 122, 12-18. [CrossRef]

25. Glayeri, A.; Rabbani, M. New Technique in Semi-Analytic Method for Solving Non-Linear Differential Equations. Math. Sci. 2011, 5, 395-404. 
26. Rabbani, M. Modified homotopy method to solve non-linear integral equations. Int. J. Nonlinear Anal. Appl. 2015, 6, 133-136.

27. Rabbani, M. New Homotopy Perturbation Method to Solve Non-Linear Problems. J. Math. Comput. Sci. 2013, 7, 272-275. [CrossRef]

28. Adomian, G. Solving Frontier Problem of Physics: The Decomposition Method; Kluwer Academic Press: Dordrecht, The Netherlands, 1994.

29. Rabbani, M.; Arab, R.; Hazarika, B. Solvability of nonlinear quadratic integral equation by using simulation type condensing operator and measure of noncompactness. Appl. Math. Comput. 2019, 349, 102-117. [CrossRef]

(C) 2019 by the authors. Licensee MDPI, Basel, Switzerland. This article is an open access article distributed under the terms and conditions of the Creative Commons Attribution (CC BY) license (http:/ / creativecommons.org/licenses/by/4.0/). 